\title{
Effect of combined tensile, bending and torsion deformation on medium carbon steel wire
}

\author{
Marina Polyakova ${ }^{1, a}$, Aleksandr Gulin ${ }^{1}$ and Eduard Golubchik ${ }^{1}$ \\ ${ }^{1}$ Nosov Magnitogorsk state technical university, Materials Processing Department, 38 Lenin Avenue, Magnitogorsk, Russia
}

\begin{abstract}
Such schemes of plastic deformation as bending, torsion, tension, compression etc. are considered to be the basic schemes for metal processing methods. Each of these types of deformation has specific influence on microstructure and mechanical properties of the processed metal. For the optimal result of plastic deformation impact on metal structure and properties the mechanism of plastic processing should follow the definite requirements. The effect of different kinds of deformation on medium carbon steel wire was studied using several methods: scanning electron microscopy, atomic force microscopy, dynamic hardness testing. The obtained results can be used in the design of combined methods of deformation processing of carbon steel.
\end{abstract}

\section{Introduction}

The main tendency in the development and design of effective engineering processes in metallurgy is considered to be the creation of new technological systems with little quantity of operations, without wastes and manual management. But at the same time improving the labour efficiency and essential increase of product quality are of high importance. Application of such technologies simplifies the structure of production process, changes the essence of technological operations and as a result decreases the quantity of equipment, material and energy sources necessary for the production $[1-4]$. At the same time it is obvious that continuous technological processes are more preferable from this point of view. Hence the competitiveness of the manufacturing process depends to high extent on the implementation the continuous technological processes at the industrial enterprises.

The technological process of metal ware production is the multioperation one and the only one operation is detected as the basic operation during which the transformation of the workpiece size and material occurs. Such kinds of deformation as tension, compression, bending and twisting form the basics of different types of metal deformational processing because they change properties and microstructure of the processed metal in a wide range. It is the perspective way for new technological processes design to combine different kinds of deformation in order to obtain the metal product with definite level of properties. But this combination have to be ground on the basic operation of the technological process. Drawing is the basic operation for wire production during which wire is processed by tension and compression. When designing the new method of combined deformation it is necessary to study the peculiarities of influence of different kinds of deformation on the wire properties [5 - 7].

Alternating bending is widely used in different metal processing technologies as intermediate operation (winding, rewinding) as independent operation (sheet stamping, descaling, flattening). In some cases alternating bending can be used as the metal processing technique with specific effect on metal structure and properties without changing the shape of the product. Distinguishing from other kinds of deformation where the total strain is limited by the workpiece sizes the total strain and the workpiece length at alternating bending practically are not limited but at the same time the process efficiency can be rather high. The influence of alternating bending on carbon steel wire and ropes properties is studied in [8 12]. Analytical models which can be used for prediction the effects of nonlinear bending of wires are presented in [12]. The obtained results can be used in practice of technologies design for manufacturing wire with different cross-sections from different materials.

Another kind of deformation which has a great effect on metal properties is torsion [13 - 18]. It is the kind of deformation when each element of the workpiece goes around the horizontal axis. Twisting is the basic operation in rope manufacturing so the study of metal properties after twisting is important in order to get this kind of metal product with high quality level. Torsion is characterized by very complicated stress-strain state which is maximum on the surface and tends to zero in the center of the steel wire. Due to the basics of metal resistance theory the transversal lines on the surface of the round workpiece after it is twisted remain plane, diameters of the cross-sections and the distances between them do not change. At the same time the rectangular net

\footnotetext{
${ }^{\text {a } C o r r e s p o n d i n g ~ a u t h o r: ~ m . p o l y a k o v a-64 @ m a i l . r u ~}$
} 
changes to the net with parallelograms. Hence, the stress state of the twisted round workpiece is considered to be the pure shear. Another factor which is necessary to take into consideration is that torsion influences the microstructure changing of carbon steel wire depending on the carbon content $[15,16]$. Experimentally it was proved that after twisting strength of the wire increased [18]. So choosing the proper modes of torsion deformation one can improve toughness and durability of the carbon steel wire.

Drawing is the main technological process for wire production. It is well known that the deformation scheme during the drawing process is characterized by longitudinal and radial stresses. Such technological parameters as die approach, bearing parts of die, drawing speed effect the mechanical properties, microstructure and texture of the processed steel wire [19 - 26]. At the same time carbon content significantly influences the properties of carbon steel wire at drawing. But drawing is considered to be rather conservative process. At present time drawing has already exhausted its resources for improving the processed metal properties by changing deformation parameters or drawing speed. So it is necessary to design new methods based on the combination of different kinds of deformation which will be very effective for production steel wire with high level of mechanical properties [27 - 29]. This way is connected with the necessity to study the peculiarities of carbon steel wire microstructure formation after different kinds of deformation.

\section{Materials and methods}

In order to estimate the possibilities of the technological constraints of the combined plastic deformation for medium carbon steel wire, the research group carried out complex research work on investigation the processing modes' impact on microstructure and mechanical properties formation [30-32]. The wire made of medium carbon steel wire containing $0.5 \% \mathrm{C}(0.5 \% \mathrm{C}-0.2 \% \mathrm{Si}-$ till $0.6 \% \mathrm{Mn}$ - till $0.25 \% \mathrm{Cu}$ - till $0.08 \% \mathrm{As}$ - till $0.25 \% \mathrm{Ni}$ - till $0.040 \% \mathrm{~S}$ - till $0.035 \% \% \mathrm{P}$ - till $0.25 \% \mathrm{Cr}$ - in wt. \%) $3.45 \mathrm{~mm}$ in diameter was chosen for the experiments. This carbon steel wire is used for a wide range of engineering components. The aim of the investigation was to identify the effect of different types of plastic deformation (reduction and elongation, bending) and their combination with torsion on medium carbon steel wire.

The deformation ratio by drawing was chosen $37 \%$ depending on the medium carbon steel wire mechanical properties in as-received state. Bending deformation was applied on steel wire while going along the moving line of the 4-roll system with rolls $90 \mathrm{~mm}$ in diameter. Torsion deformation was $150 \mathrm{rpm}$ (revolutions per minute). The combination of drawing with torsion and bending was arranged by the laboratory setup [33].

Atomic force microscopy was exercised in noncontact mode. A stiff cantilever was oscillated in the attractive regime, meaning that the tip was quite close to the sample, but not touching it. The forces between the tip and sample are quite low, on the order of $\mathrm{pN}\left(10^{-12} \mathrm{~N}\right)$. The detection scheme is based on measuring changes to the resonant frequency or amplitude of the tungsten cantilever as the interaction between the tip and sample dampen the oscillation. In order to obtain AFM images the cantilever moved along the line directly and reversly. Then it moves to the next line. The quantity of scanning points was chosen in a such way which made it possible to study the peculiarities of the carbon steel wire surface.

Scanning electron-microscope analysis of the wire in the as received state (after drawing) and after combined deformation was carried out on the electron microscope JEOL JSM-6490 LV with accelerating voltage $30 \mathrm{kV}$ in conditions of Nano Steel Research Studies Institute of Nosov Magnitogorsk state technical university. Samples for metallographic examination were prepared from the deformed wire by polishing and etching the cross-section and observed at the surface of the sample.

Microhardness was measured by Dynamic ultramicrodurometer DUH-211S. Dynamic hardness is determined by measuring an indentation depth, instead of by measuring the diagonal lengths of an indentation. Due to ISO standard (ISO14577-1), it provides for the method of requesting the following material parameters from the relation of test force and indentation depth. Martens hardness (HMT) is measured under applied test force. Martens hardness is determined from the values given by the force/indentation depth curve during the increasing of the test force, preferably after reaching the specified test force. Martens hardness includes the plastic and elastic deformation, thus this hardness value can be calculated for Vickers indenter, triangular indenter with $115^{\circ}$ tip angle (Berkovich indenter). Martens hardness, determined from the slope of the increasing force / indentation depth curve $\left(\mathrm{HM}_{\mathrm{S}}\right)$. This hardness $\left(\mathrm{HM}_{\mathrm{S}}\right)$ has the advantage of avoiding the zero-point determination for homogeneous materials. Indentation hardness $\left(\mathrm{H}_{\mathrm{it}}\right)$ is a measure of the resistance to permanent deformation or damage. A simple function may be derived for a perfect Vickers geometry indenter, or for a Vickers indenter where the projected area function is known. In this case measurements of hardness, $\mathrm{H}_{\mathrm{IT}}$ are related to the Vickers Hardness numbers, $\mathrm{HV}^{*}$, by a scaling factor.

\section{Results and discussion}

Results of AFM of carbon steel wire after different kids of deformation processing is shows in Figure 1. Using computer visualization $2 \mathrm{D}$-images were obtained. At 2Dvisualisation each point on the surface $Z=f(x, y)$ is identified by the definite colour depending on the point height (z-coordinate).

Microstructure of medium carbon steel wire consists of ferrite-carbide composition and low quantity of structured free ferrite which is located separately along the boundaries of pearlite colonies (Figure 2). This is characteristic to such carbon content in steel. The microstructure is uniform.

After drawing one can observe that cementite plates become curved in both directions (Figure 2, a, b). 


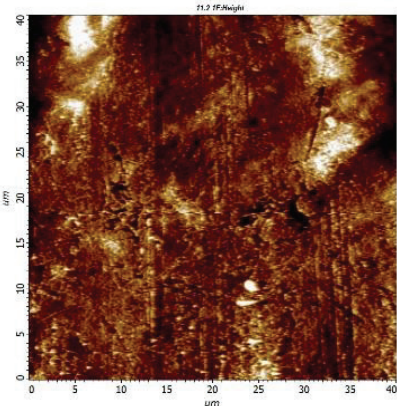

a

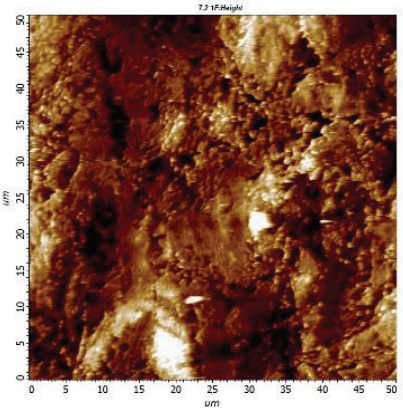

$\mathrm{d}$
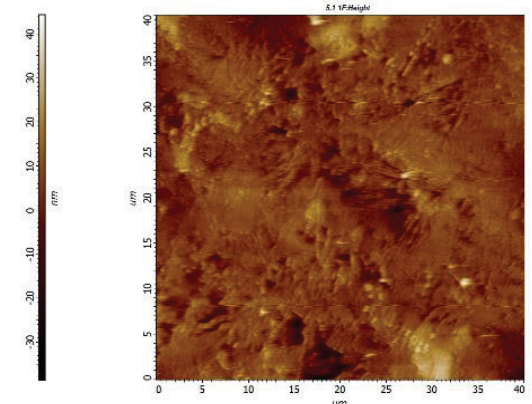

b
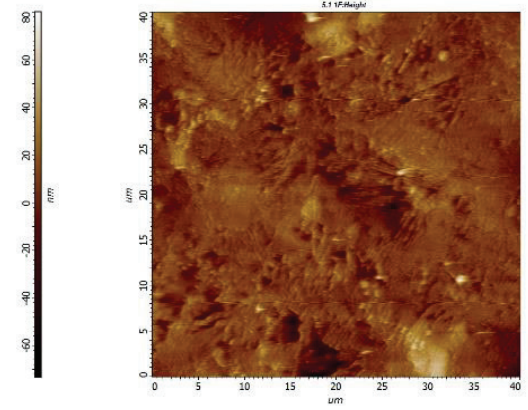

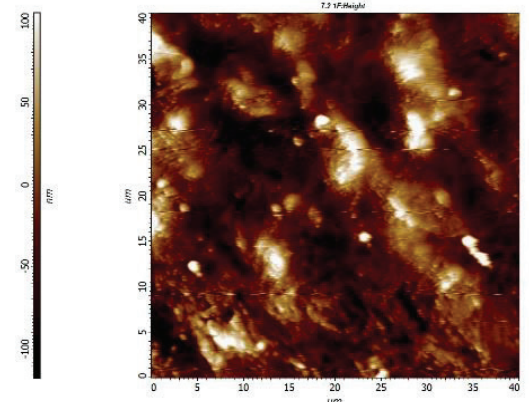

c
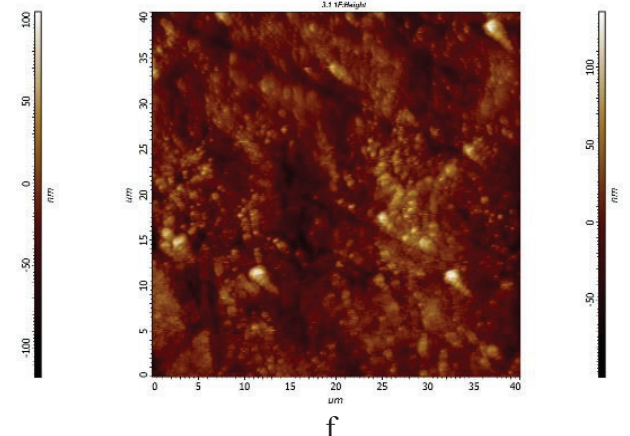

Figure 1. AFM of medium carbon steel wire after drawing (a, d), drawing with bending (b, e) and drawing with bending and torsion ( $\mathrm{c}, \mathrm{f})(\mathrm{a}-\mathrm{c}$ - transverse cross-section, $\mathrm{d}-\mathrm{f}$ - longitudinal cross-section)

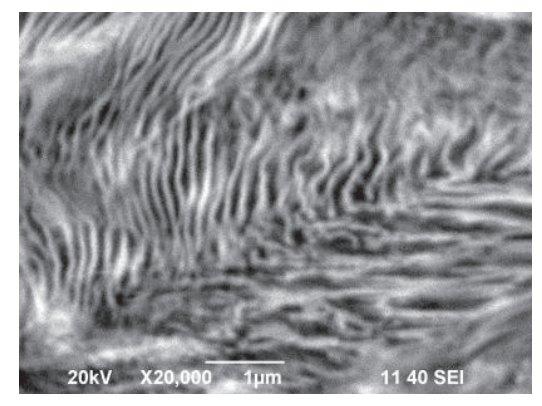

a

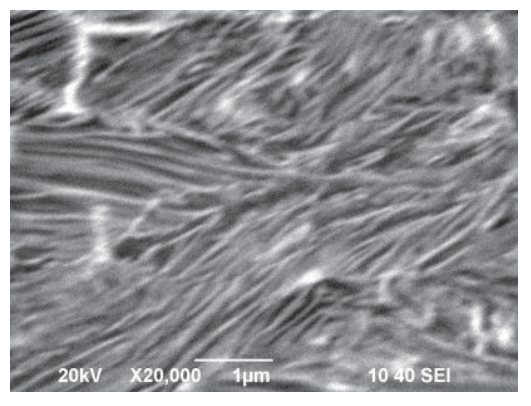

c

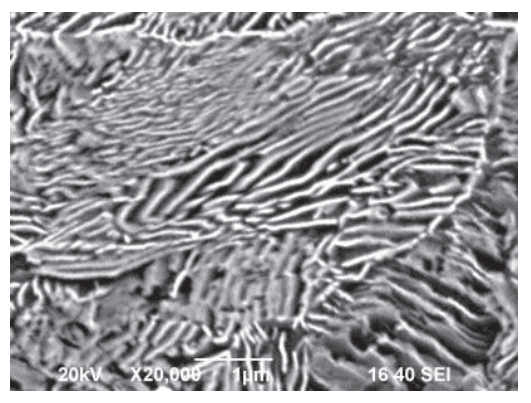

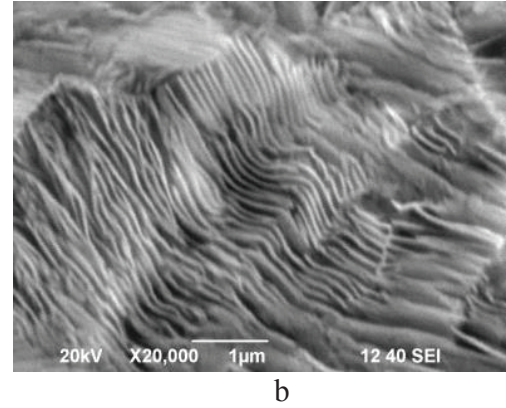
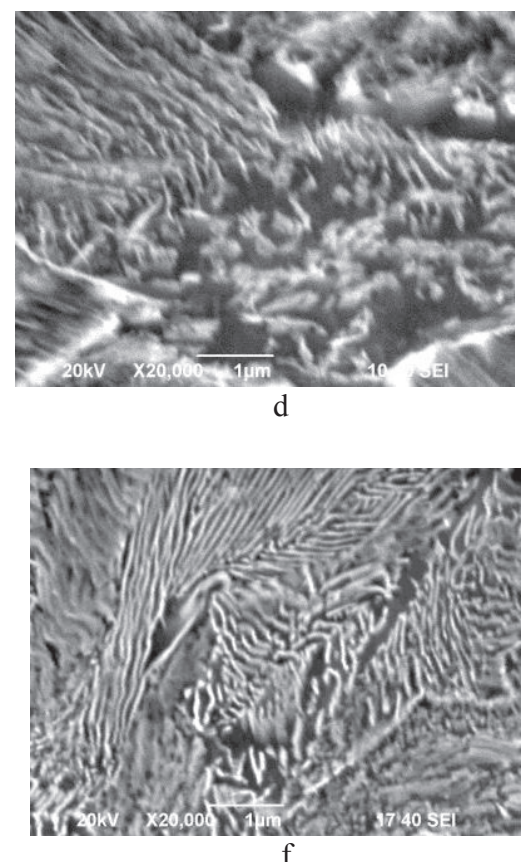

$\mathrm{f}$

Figure 2. Deformation and crashing of cementite plates after drawing $(a, b)$, drawing with bending $(c, d)$ and drawing with bending and torsion (e, f) ( $\mathrm{a}, \mathrm{c}, \mathrm{e}-$ longitudinal cross-section, $\mathrm{b}, \mathrm{d}, \mathrm{f}$ - transverse cross-section) 
Combination of drawing with bending leads to crashing the cementite plates (Figure 2, c, d) which becomes drastically after drawing with bending and torsion (Figure 2, e, f). This can be explained by the metal complex deformation processing in such deformation action. Such microstructure changes of medium carbon steel wire prove the results of dynamic hardness testing (Figure 3).

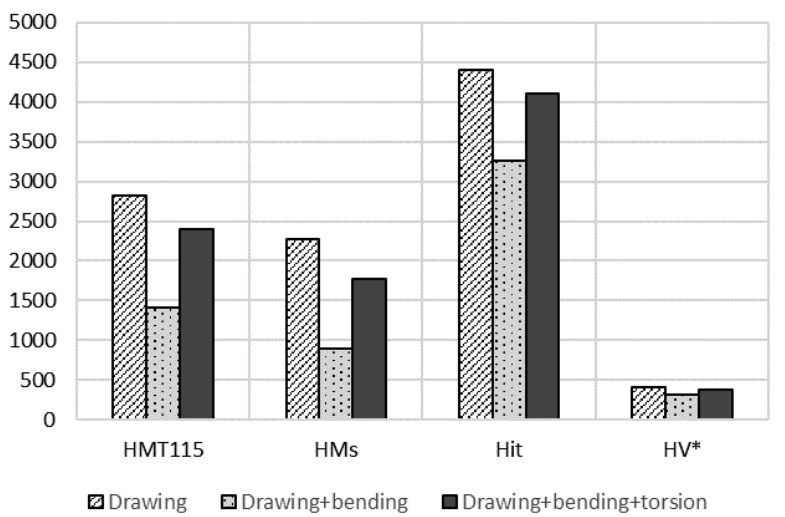

Figure 3. Dynamic hardness parameters of medium carbon steel wire after different kinds of deformation processing

As a result of combination of different kinds of deformation on medium carbon steel wire one can see the decrease of all dynamic hardness parameters. It can be explained that after such kind of deformation the medium carbon steel wire becomes more ductile but at the same time its strength does not decrease so much as compared with drawing (see Figure 3). Such peculiarities of mechanical properties which medium carbon steel wire possesses after combined deformation processing can be very useful for practical application in cable manufacturing.

\section{Conclusions}

Improving properties of carbon steel wire is the important item for investigations. The aim of practical interests is the combination of drawing as the basic operation in wire manufacturing with other kinds of deformation. The presented investigation shows the effect of different deformational processing on the medium carbon steel wire microstructure and dynamic hardness changing. As a result of combined deformational processing the medium carbon steel wire becomes more ductile as compared with it after drawing. Experimental results of the peculiarities of medium carbon steel wire after different kinds of combined deformational processing can be used in wide range of practical applications where it is used both as a finished product and as an intermediate workpiece.

\section{References}

1. H-S. Park, N-H. Tran. Int J Adv Manuf Tech. 56 (912) $1159-1165$ (2011).

2. H-S Park, N-H. Tran. Int J Control, Automation and Systems. 10 (4) 806-816 (2012).
3. M.B. Sokol. J of Business and Psychology. 8 (3) 277-296 (1994).

4. N. Kim, J.H. Pae. J of the Academy of Marketing Science. 35 (2) 259-269 (2007).

5. Saunders, J. Nutting. Met Sci. 18 (12) 571-575 (1984)

6. Verpoest, B.D. Notohardjono, E. Aernoudt. ASTM Spec Tech Publ. 361-377 (1985)

7. M. Zelin, R.M. Shemenski. Conf. Proc. Wire Assoc. Int., Inc.: Wire Expo. 1-14 (2006)

8. R. Kruzel. Metalurgija. 52 (1) 93-95 (2013)

9. G. Oplatka. Proc 9th Cong Mater Test. 44-48 (1986)

10. D. Abel. Wire. 52 (1) 24-25 (2002)

11. M. Di Ludovico, G.P. Lignola, A. Prota, E. Cosenza. ACI Struct J. 107 (4) 390-399 (2010)

12. S. Baragetti. Meccanica. 41 443-458 (2006)

13. Blackmore. Wire J Int. 39 (4) 98-102 (2006)

14. J. Hajduk. Arch Budowy Masz. 32 (3-4) 161-173 (1985)

15. N. Guo, B. Song, B-S. Wang, Q. Liu. Acta Metall. Sin. 28 (6) 707-714 (2015)

16. C. Cordier-Robert, B. Forfert, B. Bolle, J-J. Fundenberger, A.Tidu. J Mater Sci. 43 1241-1248 (2008)

17. B. Goes, A. Martin-Meizoso, J. Gil-Sevillano, I. Lefever, E. Aernoudt. Eng Frac Mech. 60 (3) 255272 (1998)

18. F. Knap. Wire World Int. 29 (4) 94-96 (1987)

19. R.L. Plaut, A.F. Padilha, N.B. Lima, C. Herrera, A.F.Filho, L.H.Yoshimura. Mater Sci and Eng A, 499 337-341 (2000)

20. F. Fang, X-j. Hu, B-m. Zhang, Z-h. Xie, J-q. Jiang. Mater Sci and Eng A. 583 78-83 (2013)

21. J. Adamczyk, M. Suliga, J.W. Pilarczyk, M. Burdek. Arch Metal and Mater 57 (4) 1105-1110 (2012)

22. J.W. Pilarczyk, J. Krnac, J. Adamczyk, B. Golis. Conf. Proc. Annu. Conf. Wire Assoc. Int. (2011)

23. J. Krnac, R. Wludzik, J.W. Pilarczyk, R. Kruzel. Conf. Proc. Wire Assoc. Int., Inc. - Wire Cable Tech. Symp., Annu. Conf. 163-176 (2009)

24. M. Zidani, S. Maessaoudi, T. Baudin, D. Solas. M.H. Mathon. Int J Mater Form 3 7-11 ( 2010)

25. M. Suliga. Arch Metal and Mater 57 (4) 1021-1030 (2012)

26. Nemec, B. Golis, J.W. Pilarczyk, R. Budzik, W. Waszkielewicz. Conf. Proc. Wire Assoc. Int., Inc.: Wire Expo 182-197 (2006)

27. M. Zelin, R.M. Shemenski. 2006 Conference Proceedings of the Wire Association International. 1-4 (2006).

28. M. Chukin, A. Korchunov, M. Polyakova, D. Emaleeva. Steel in Translation. 40 (6) 595-597 (2010).

29. M. Polyakova, E. Golubchik, Yu. Efimova, A. Gulin. Key Eng Mater. 724 77-86 (2017).

30. Calliari I., Brunelli K., Polyakova M., Gulin A., Golubchik E. 5th Int Conf TMP 2016.

31. M.V. Chukin, M.A. Polyakova, A.E. Gulin. Steel in Translation. 46 (8) 548-551 (2016).

32. M. Polyakova, I. Calliari, A. Gulin. Key Eng Mater. 716 201-207 (2016). 
33. M. Polyakova, M. Chukin, E. Golubchik, A. Gulin, RU Patent 130,525. (2013) 